\title{
Linear Approximation to Optimal Control Allocation for Rocket Nozzles With Elliptical Constraints
}

\author{
Jeb S. Orr* \\ Science Applications International Corporation, Huntsville, AL, 35806 \\ John W. Wall ${ }^{\dagger}$ \\ Dynamic Concepts, Inc., Huntsville, AL, 35806
}

\begin{abstract}
In this paper we present a straightforward technique for assessing and realizing the maximum control moment effectiveness for a launch vehicle with multiple constrained rocket nozzles, where elliptical deflection limits in gimbal axes are expressed as an ensemble of independent quadratic constraints. A direct method of determining an approximating ellipsoid that inscribes the set of attainable angular accelerations is derived. In the case of a parameterized linear generalized inverse, the geometry of the attainable set is computationally expensive to obtain but can be approximated to a high degree of accuracy with the proposed method. A linear inverse can then be optimized to maximize the volume of the true attainable set by maximizing the volume of the approximating ellipsoid. The use of a linear inverse does not preclude the use of linear methods for stability analysis and control design, preferred in practice for assessing the stability characteristics of the inertial and servoelastic coupling appearing in large boosters. The present techniques are demonstrated via application to the control allocation scheme for a concept heavy-lift launch vehicle.
\end{abstract}

\section{Introduction}

The use of optimal control allocation has received extensive attention in the literature, particularly in the context of aircraft control. ${ }^{1,2}$ Typically, aircraft are designed with significantly overlapping control moment effectiveness at a particular flight condition, so the resultant control allocation problem is underdetermined. Likewise, in the context of launch vehicle dynamics, the development of large, heavy-lift rockets has led to renewed interest in the applications of optimal control allocation schemes to systems with multiple gimbaled rocket nozzles.

In order to enhance performance margins, crew safety, and likelihood of mission success in the event of severe internal and external disturbances, it is desirable to design a control allocation mechanism that is capable of effecting the maximum possible angular accelerations on the vehicle while minimizing the opportunity for saturation of the control actuators. However, straightforward techniques such as daisy chaining and cascaded inverses ${ }^{3}$ are undesirable since they are fundamentally a function of the magnitude and direction of the applied angular acceleration command; the resultant nonlinearity precludes the use of linear stability analysis techniques, ${ }^{4}$ often considered mandatory for humanrated launch systems. Unlike in some aircraft where actuator servoelastic coupling and inertial reaction torques may be ignored under the premise that the control allocation algorithm provides exactly the required angular acceleration, these effects are non-negligible and sometimes dynamically significant for large flexible rocket vehicles. ${ }^{4,5}$ The use of multiple engines with varied low-frequency servodynamics requires that the exact combination of actuators to be employed at any flight condition is known; for stability analysis, it is insufficient to assume that the angular acceleration command is automatically achieved by the control allocator in light of the dynamics affecting the motion of applied thrust and the inertial coupling of the relatively heavy nozzles themselves.

In the case of gimbaled rocket engines, the maximum control deflection is limited by the linear travel of the actuators, which are often of the high-power hydraulic type. The nozzles of liquid-fueled motors are usually suspended from a two-degree of freedom gimbal hinge attached forward of the thrust chamber; in the case of a solid-fueled motor, a flexible bearing is used. The actuators are applied in pairs spaced 90 degrees apart about the thrust vector to effect local pitch and yaw deflection of the engine bell. It is sufficient in either case to model the thrust force as a twodegree-of-freedom vector pivoting in space, with the thrust force applied at the pivot point. Neglecting losses due to the geometry of the linkage between the linear actuator and the angular motion of the nozzle, the motion is constrained approximately within a rectangular boundary. Additional constraints, such as clearance among adjacent nozzle bells and/or thrust structure, may further limit the allowable nozzle motion.

${ }^{*}$ Flight Controls Engineer, Space Engineering Technology Division

${ }^{\dagger}$ Engineer, Guidance, Navigation, and Control Group 
Multi-actuated thrust-vector control systems of this type are common in large launch vehicles, and are in fact (for the unconstrained case) almost an ideal benchmark problem for control allocation given that most multiple-nozzle configurations yield redundant control authority in all three control axes. If the thrust vectors are symmetric and nominally aligned along the body thrust axis (Figure 1), the allocation problem is near trivial owing to the obvious thrust vector mixing that must occur in order to generate separate roll, pitch, and yaw accelerations. However, if the nozzles are asymmetrically located and canted with respect to the control axes (Figure 2), the solution of the control allocation problem that generates the required accelerations is not straightforward.

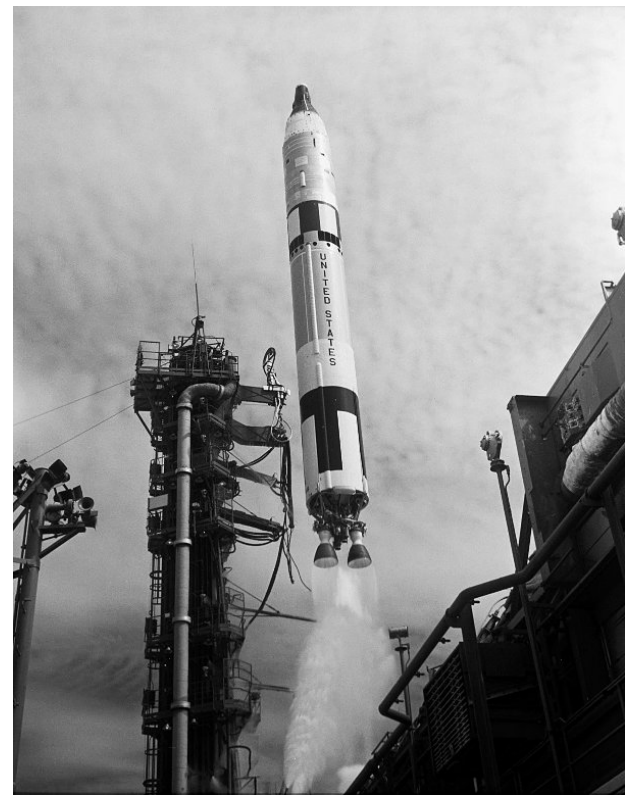

Figure 1. Symmetric actuation; Gemini-Titan launch vehicle (Image credit: NASA)

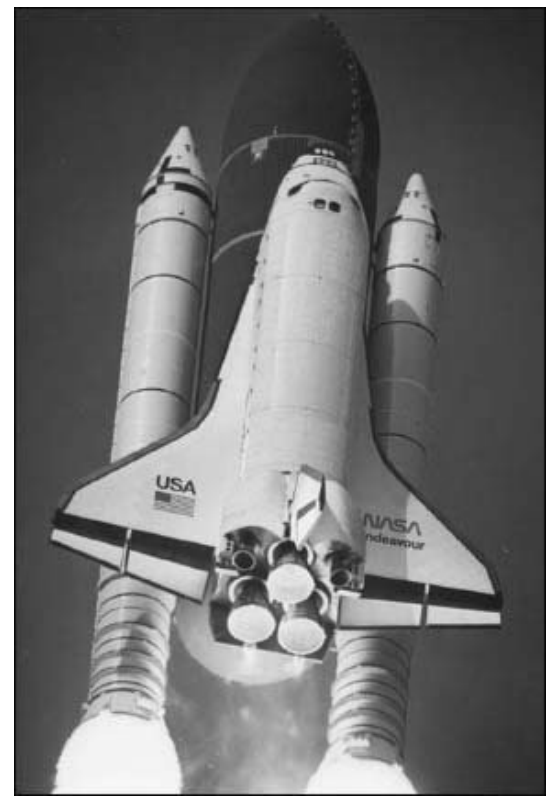

Figure 2. Asymmetric actuation; Space Shuttle launch vehicle (Image credit: $N A S A)$

Reaching the hardware saturation limits is undesirable due to potential damage to the actuator and/or the thrust structure, so the actuator command is usually software-limited to a value slightly less than the hardware boundary. The implementation of the software limits may take into account the potentially complex constraint boundaries, or may adopt the simplified approach of applying a root-sum-square angle constraint to the nozzle deflection. It is often convenient, and perhaps optimal, to constrain nozzle motion to an elliptical boundary, especially in order to mitigate nozzle bell interference. In the present research, we are concerned with the case where nozzle travel of any actuator pair is limited by a constraint boundary prescribed as an ellipse normal to the undeflected thrust direction. The thrust directions are defined as small rotations about the 2,3 axes of a coordinate frame whose 1-axis is aligned with the undeflected thrust.

Much of the existing literature on control allocation ${ }^{1,6}$ treats extensively the case where the applied moment is linear in the controls and the actuator saturation constraints are independent and symmetric with respect to a single actuator degree of freedom $\eta$; that is,

$$
|\eta| \leq 1 .
$$

In our application, the moment is linear in the controls for small deflections, but each actuator constraint function $c_{i}$ applies to two degrees of freedom, such that

$$
\delta_{i}=\left[\begin{array}{ll}
\eta_{i}^{1} & \eta_{i}^{2}
\end{array}\right]^{T}, \quad c_{i}\left(\delta_{i}\right) \leq 1 .
$$

One particularly difficult problem in the design of control allocators is the determination of the geometry of the set of attainable angular accelerations that are achievable using a particular allocation strategy. Efficient numerical methods that handle single-degree-of-freedom constraints have been developed extensively, but no such techniques exist for elliptically-constrained systems. As will be shown, the convenient properties of the calculus of ellipsoids ${ }^{7}$ can be used 
to rapidly and efficiently estimate the geometry of the attainable control accelerations. The resultant approximation can then be used in the formulation of a computationally superior objective function for rapid numerical optimization with respect to a parameterized allocation mechanism.

\section{Control Allocation With Elliptical Constraints}

\section{A. Control Mapping for Multiactuated Rockets}

The general linear control allocation problem is concerned with the mapping of the deflections of $m$ actuators into an $n$-dimensional moment space, and the relationship of a closed, bounded, and convex set $\Omega$ of admissible control deflections in $\mathbb{R}^{m}$ to its associated closed, bounded, and convex set of achievable moments $\Phi$ in $\mathbb{R}^{n}$. In this application, we consider an $m=2 k$-dimensional control deflection space, consisting of $k$ nozzles each with two degrees of freedom, and its relationship to a $n=3$-dimensional moment space ${ }^{\ddagger}$ of instantaneous angular accelerations about some fixed set of body axes of a rigid body.

Consider the Euler equations of rigid body motion given by

$$
J \dot{\omega}+\omega^{\times} J \omega=g
$$

where $\omega$ are the instantaneous rates about a body-axes frame fixed at the center of mass; $J$ is an appropriate inertia tensor, and $g \in \mathbb{R}^{3}$ is the applied torque. Let the torque be given by an affine linear transformation of the control vector $\Delta$

$$
g=\sigma+M \Delta
$$

where $M$ is the moment matrix, $\sigma$ is the residual, and

$$
\Delta=\left[\begin{array}{llll}
\delta_{1}^{T} & \delta_{2}^{T} & \ldots & \delta_{k}^{T}
\end{array}\right]^{T}
$$

consists of the angular deflection vectors $\delta_{i} \in \mathbb{R}^{2}$ over $k$ actuators, so $\Delta \in \mathbb{R}^{2 k}$ and $M \in \mathbb{R}^{3 \times 2 k}$. We have assumed that the applied moment is linear and symmetric in the controls $\Delta$.

Consider the nozzle and constraint geometry shown in Figure 3. For a rocket nozzle with scalar thrust $F_{i}$ oriented nominally along a body frame unit vector $u_{i}$, the moment contribution due to an actuator deflection is given by

$$
g_{i}=r_{i}^{\times}\left[T_{\theta}\right] F_{i} u_{i}
$$

where $\left[T_{\theta}\right]$ is the kinematic transformation relating the nozzle thrust direction to the undeflected thrust direction unit vector. If the rotation is small, $\left[T_{\theta}\right]$ can be expanded using the skew-symmetric small rotator and

$$
g_{i}=r_{i}^{\times} F_{i} u_{i}-F_{i} r_{i}^{\times} u_{i}^{\times} \theta_{i}
$$

where $\theta_{i} \in \mathbb{R}^{3}$ is a vector of small rotations. The former component is moment due to undeflected thrust and the latter is a linear function of the change in thrust direction $\theta_{i}$. It follows that the net torque can be expressed as

$$
\sigma+M \Delta=\sum_{i=1}^{k} r_{i}^{\times} F_{i} u_{i}-\sum_{i=1}^{k} F_{i} r_{i}^{\times} u_{i}^{\times}\left[T_{i}^{B G}\right] \delta_{i}
$$

if $\left[T_{i}^{B G}\right] \in \mathbb{R}^{3 \times 2}$ is a partial transformation that maps the rotations $\delta_{i}$ in gimbal frame coordinates $(G)$ into the body frame.

\footnotetext{
${ }^{\ddagger}$ For numerical and practical convenience, the space $\Phi$ is that of angular accelerations in $\mathbb{R}^{3}$. For consistency with the literature, we will refer to this as a moment space.
} 


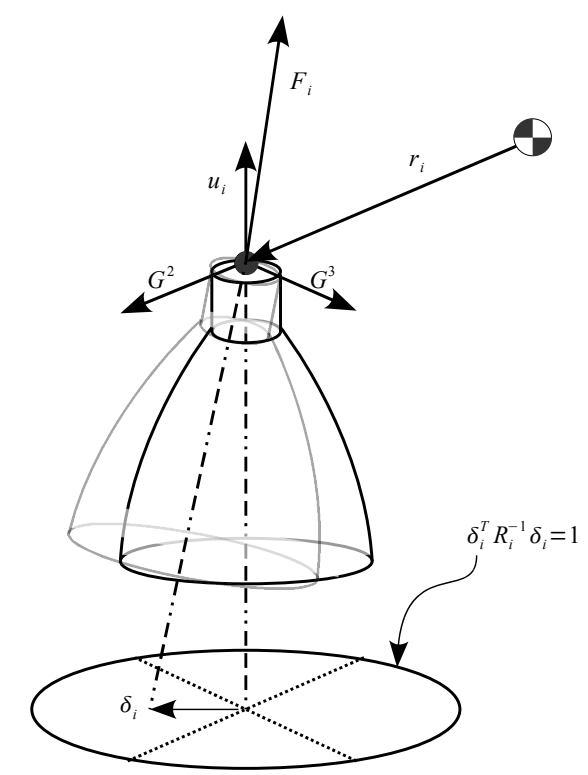

Figure 3. Nozzle and constraint geometry

It is possible to block diagonalize the $\left[T_{i}^{B G}\right]$ and express $M$ as

$$
M=\left[\begin{array}{llll}
M_{1} & M_{2} & \ldots & M_{k}
\end{array}\right]\left[T^{B G}\right]=\left[\begin{array}{llll}
-F_{1} r_{1}^{\times} u_{1}^{\times} & -F_{2} r_{2}^{\times} u_{2}^{\times} & \ldots & -F_{k} r_{k}^{\times} u_{k}^{\times}
\end{array}\right]\left[T^{B G}\right] .
$$

We are interested in the effects of perturbations in the $\delta_{i}$ on the angular acceleration $\dot{\omega}$. To that end, we linearize (3) about $\omega_{0}=0$ and solve for $\dot{\omega}$ to find

$$
\dot{\omega}=J^{-1} \sigma+J^{-1} M \Delta=\rho+B \Delta=\Gamma .
$$

Without loss of generality, we assume that there exists a set of $u_{i},\left[T_{i}^{B G}\right]$ such that $\rho=0$; that is, there is a trim solution of vehicle nozzle cant angles at each flight condition that provides no residual angular acceleration. Thereafter, we consider only the linear mapping $\Gamma=B \Delta$, and as in (9), we partition $B$ such that

$$
B=\left[\begin{array}{llll}
B_{1} & B_{2} & \ldots & B_{k}
\end{array}\right]
$$

and each $B_{i} \in \mathbb{R}^{3 \times 2}$ so we can also write $\Gamma=\sum \gamma_{i}$, where $\gamma_{i}=B_{i} \delta_{i}$.

Finally, we suppose that the maximum travel of each control vector $\delta_{i}$ is bounded by the elliptical constraint

$$
\delta_{i}^{T} R_{i}^{-1} \delta_{i} \leq 1, R_{i}=R_{i}^{T}>0
$$

where $R_{i} \in \mathbb{R}^{2 \times 2}$ is the shape matrix of the constraint ellipsoid $\mathcal{E}\left(R_{i}\right)$, and is a symmetric positive definite matrix. In most cases, the axes of the constraint ellipse described by (11) are aligned with the actuator deflection axes, so $R_{i}$ is diagonal, and the lengths of the major and minor axes are given by the square root of the diagonal entries (eigenvalues) of $R_{i}^{-1}$.

\section{B. Topology of the Constraints}

In defining a constraining ellipsoid $\mathcal{E}_{i}\left(R_{i}\right)$ associated with each $\delta_{i}$, we have defined a closed and bounded convex set $\xi_{i}$ for each $\delta_{i}$ given by

$$
\xi_{i}=\left\{\delta_{i} \in \mathbb{R}^{2} \mid \delta_{i}^{T} R_{i}^{-1} \delta_{i} \leq 1\right\} .
$$

The admissible controls $\xi_{i}$ are $k$ subsets of $\mathbb{R}^{2}$ or equivalently a convex subset $\Omega$ of $\mathbb{R}^{m}$. Each $\xi_{i}$ has associated with it an image in $\mathbb{R}^{3}$ given by the mapping $B_{i}$. Necessarily, the image of $\xi_{i}$ in $\mathbb{R}^{3}$ is a degenerate ellipsoid (an ellipse) that 
is symmetric about the origin (under the assumption $\rho=0$ ) and whose principal axes are oriented with respect to a particular basis in $\mathbb{R}^{3}$ by some linear transformation. Each $B_{i}$ can be used to determine the associated shape matrix $Q_{i}$ that describes the image of $\xi_{i}$ in $\mathbb{R}^{3}$, an ellipsoid $\mathcal{E}\left(Q_{i}\right)$ given by

$$
\Xi_{i}=\left\{\gamma_{i} \in \mathbb{R}^{3} \mid \gamma_{i}^{T} Q_{i}^{-1} \gamma_{i} \leq 1\right\} .
$$

Let us assume that for each set $\xi_{i}$ and its associated map $B_{i}$, there exists a unique inverse shape matrix $Q_{i}^{-1}$ such that

$$
\delta_{i}^{T} R_{i}^{-1} \delta_{i}=\gamma_{i}^{T} Q_{i}^{-1} \gamma_{i}=1 .
$$

That is, for each admissible control deflection $\delta_{i}$ that is on the constraint boundary, there is an associated $\gamma_{i}=B_{i} \delta_{i}$ such that $\gamma_{i}^{T} Q_{i}^{-1} \gamma_{i}=1$. Suppose that $P_{i}^{\star}$ is the unique generalized inverse of $B_{i}$ that satisfies the Moore-Penrose conditions ${ }^{8}$ such that $\mathcal{R}\left(P_{i}^{\star T}\right)=\mathcal{R}\left(B_{i}\right)$. We therefore have excluded $\mathcal{R}\left(B_{i}\right)^{\perp}=\mathcal{N}\left(B_{i}^{T}\right)$ in computing $P_{i}^{\star 9}$, and $P_{i}^{\star}$ satisfies $B_{i} P_{i}^{\star}=I$. Letting $\delta_{i}=P_{i}^{\star} \gamma_{i}$, we find from (13) that

$$
\gamma_{i}^{T} P_{i}^{\star T} R_{i}^{-1} P_{i}^{\star} \gamma_{i}=\gamma_{i}^{T} Q_{i}^{-1} \gamma_{i}
$$

and it follows that the shape matrix

$$
Q_{i}^{-1}=P_{i}^{\star T} R_{i}^{-1} P_{i}^{\star} .
$$

One also observes that the direction in $\mathbb{R}^{3}$ normal to the degenerate ellipsoid $\Xi_{i}$ is in the null space of $B_{i}^{T}$, and $\mathcal{N}\left(B_{i}^{T}\right)=\mathcal{N}\left(Q_{i}^{-1}\right)$. Also, since $\mathcal{E}\left(Q_{i}\right)$ is degenerate, $Q_{i}^{-1}$ is singular and thus $Q_{i}$ is ill-defined.

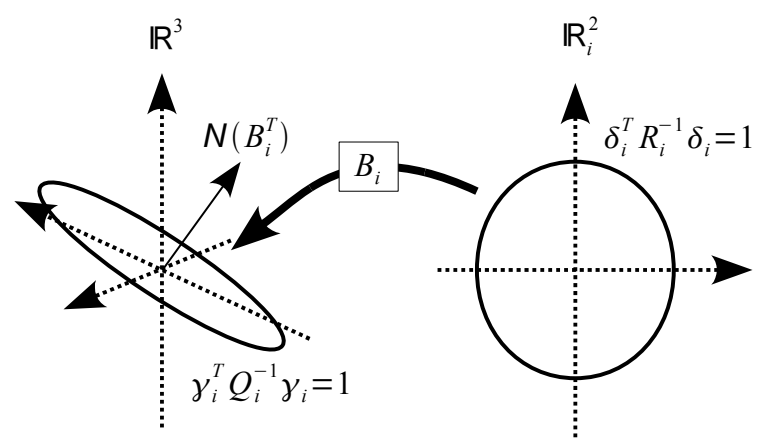

Figure 4. Topology of constraints in $\mathbb{R}^{3}$ and $\mathbb{R}^{2}$

Recall from (8) that the moments are linear in the controls and symmetric about the origin. It follows that the attainable moment set (AMS), denoted by $\Phi$, can be formed from the Minkowski sum of the $\Xi_{i}$, the geometric sum of the images of the admissible controls $\xi_{i}$ under the transformations $B_{i}$;

$$
\Phi=\Xi_{1} \otimes \cdots \otimes \Xi_{k}=\bigcup_{\gamma_{1} \in \Xi_{1}} \cdots \bigcup_{\gamma_{k} \in \Xi_{k}}\left\{\gamma_{1}+\cdots+\gamma_{k}\right\} .
$$

The AMS $\Phi$ is therefore the sum of $k$ degenerate ellipsoids in $\mathbb{R}^{3}$, yielding, if $\operatorname{rank}(B)=3$, a volumetric closed, bounded, and convex set. The set $\Phi$ is not, in general, an ellipsoid, but is bounded by an arbitrary (not necessarily smooth) manifold $\partial(\Phi)$. We note that the boundary of the set computed from geometric sum (14) is exactly the image in $\mathbb{R}^{3}$ under the linear map $B$ of the $m$-dimensional smooth manifold $\partial(\Omega)$ bounding the controls $\Delta$.

\section{Linear Inverses}

The linear control allocation problem is concerned with determining a suitable generalized inverse $P$ for $B$; we desire a control vector $\Delta$ such that the angular acceleration is prescribed, that is, $\Gamma=\dot{\omega}_{c}$, and

$$
\Delta=P \dot{\omega}_{c} .
$$

American Institute of Aeronautics and Astronautics 
We are concerned with the class of commands $\dot{\omega}_{c}$ such that a solution exists where $\Gamma=\dot{\omega}_{c}$ and no constraints on the actuator motion are violated. The control allocation problem can be expressed as the constrained minimization that is well-addressed through a least squares procedure. If a solution does not exist within the constraint boundaries such that $\Gamma=\dot{\omega}_{c}$, it is often tempting to pursue least squares methods that minimize the norm $\left\|\dot{\omega}_{c}-B \Delta\right\|_{2}$ subject to the constraints, but one often is led to a minimum norm solution that does not satisfy the additional condition that the achieved angular acceleration is collinear with the commanded angular acceleration. This situation causes undesirable coupling of the control axes and is a nonlinear inverse with respect to the commanded acceleration. This type of solution is avoided in the present application by scaling the angular acceleration command to lie within the attainable moment set, if the geometry of the attainable moment set is known.

The linear control allocator is in general a right generalized inverse $P$ such that $B P=I$. If $B$ is not rank deficient and the number of effector degrees of freedom $m=2 k>n$, the system is underdetermined and a solution is not unique. In the most general case, the minimum number of parameters that uniquely determine the inverse $P$ is $(m-n) n^{1}$, and each of these parameterized family of linear inverses has associated with it a unique subset of the AMS which we will denote $\Phi^{\star}$. We refer to the subset $\Phi^{\star}$ as the attainable moment subset under a particular transformation. The set $\Phi^{\star}$ is a proper subset of $\Phi$; the map $B$ (and the associated family of right inverses $P$ ) are linear transformations that map a point in the control deflection set $\Omega$ onto $\partial(\Phi)$ or into its interior. This leads to the following theorem.

Theorem. Given the linear map $\Gamma=B \Delta, \Gamma \in \mathbb{R}^{n}, \Delta \in \mathbb{R}^{m}, m>n$, a compact, convex geometric constraint $\Delta \in \Omega$, and any linear allocator $P$ satisfying $B P=I$, the associated particular AMS $\Phi^{\star} \subsetneq \Phi$, a proper subset of the total AMS $\Phi$.

Proof. The following proof is similar to that given by Durham ${ }^{1}$ and is fundamental in the linear allocation problem. Consider the subset of controls $\Psi=\{\Delta \in \partial(\Omega)\}$ that lie on the boundary $\partial(\Omega)$. Since $\Omega$ is bounded, the span of $\Psi$ is necessarily $m$. Suppose that $\Phi^{\star}=\Phi$, then $B \Delta \in \partial(\Phi)$ and $P \Gamma=P B \Delta \in \partial(\Omega) \forall \Delta \in \Psi$, so $\Delta=P B \Delta \Rightarrow(P B-I) \Delta=0$ $\forall \Delta \in \Psi$. It follows that $\Psi=\{\Delta \in \Omega \cap \mathcal{N}(P B-I) \mid B \Delta \in \Phi\}$. If this is true, $\operatorname{span}(\mathcal{N}(P B-I))=m \Rightarrow P B=I$, which cannot hold if $m>n$, a contradiction.

A key consequence of the above theorem is that there is no inverse $P$ that maps every point on $\partial(\Omega)$ to $\partial(\Phi)$. Intuitively, the null space of $(P B-I)$ (those controls that contribute to the boundary of $\left.\Phi^{\star}\right)$ is precisely the orthogonal complement of the null space of $B$ (those controls that do not affect $\Gamma$ ). Every particular inverse $P$ allows access to some subset of $\Phi$, and a plausible design goal of a control allocator is to shape the particular inverse to make this subset as large as possible. The true shape of $\Phi^{\star}$ under elliptical constraints is difficult to determine directly. It can be analyzed numerically using a bisection search along unique gridded directions on the unit ball, each yielding a maximum attainable command magnitude in particular direction. The total geometry is then found using the convex hull of these gridpoints, with accuracy increasing as the number of unique directions increases. This technique is effective but extremely computationally intensive, and is not well-suited to forming a scalar objective function for optimization. Instead, ellipsoidal techniques can be applied to approximate $\Phi$ and $\Phi^{\star}$.

\section{Approximation of Attainable Moment Set}

It has been shown that the AMS can be approximated optimally by a convex set inscribing it, ${ }^{2}$ and the concept of an ellipsoidal approximation to the AMS has also been discussed, although only via a graphical interpretation. ${ }^{10}$ Determination of a single candidate approximating ellipsoid that well-describes $\Phi$ can be determined as one of a family of tight approximating ellipsoids $\mathcal{E}(Q)$ parameterized by the vector $l \in \mathbb{R}^{n}$. The approximation

$$
\mathcal{E}(Q[S(k)]) \subseteq \sum_{i=1}^{k} \mathcal{E}_{i}\left(Q_{i}\right)
$$

with

$$
Q[S(k)]=\left(\sum_{i=1}^{k} S_{i} Q_{i}^{1 / 2}\right)^{T}\left(\sum_{i=1}^{k} S_{i} Q_{i}^{1 / 2}\right)
$$

and the orthogonal matrices

$$
S(k)=\left\{S_{1}, \ldots, S_{k}\right\}, S_{i}^{T} S_{i}=I
$$


is a tight approximation along direction $l$ if its support ${ }^{11}$

$$
\left(\rho(l \mid \mathcal{E}(Q[S]))^{2}=\left(\sum_{i=1}^{k}\left(l^{T} Q_{i} l\right)^{1 / 2}\right)^{2} .\right.
$$

A tight approximation is possible along the unit vector $l \in \mathbb{R}^{3}$ if the vectors $S_{1} Q_{1} l, S_{2} Q_{2} l, \ldots, S_{k} Q_{k} l$ are aligned, that is, if there exist $k_{i j}$ such that

$$
S_{i} Q_{i}^{1 / 2} l=k_{i j} S_{j} Q_{j}^{1 / 2}, k_{i j}=k_{j i}^{-1}
$$

or equivalently, that the matrices $S_{i}$ are such that $S_{i} Q_{i}^{1 / 2} l=\lambda_{i} p$ for some vector $p \in \mathbb{R}^{n} \neq 0$. By varying $l$ over the unit ball, we can map out the entirety of $\partial(\Phi)$; each internal approximation is tangent to the manifold at a minimum of two points (along $\pm l$ ). If a single approximation is to be chosen, it is associated with a particular direction line. Given a suitable choice of the direction of approximation $l$, we must compute the matrices $S_{i}$ to satisfy (16). Let $S_{1}=I$,

$$
\begin{aligned}
v_{i} & =Q_{i} l \\
w_{i} & =Q_{i+1} l
\end{aligned}
$$

and normalize $v_{i}, w_{i}$ as $\hat{v}_{i}=v_{i} /\left|v_{i}\right|, \hat{w}_{i}=w_{i} /\left|w_{i}\right|$. We then compute a singular value decomposition ${ }^{12}$ (SVD) such that

$$
\begin{aligned}
v_{i} & =U_{v} \Sigma_{v} V_{v}^{T} \\
w_{i} & =U_{w} \Sigma_{w} V_{w}^{T}
\end{aligned}
$$

and a suitable transformation is found from

$$
S_{i+1}=U_{w} V_{w} V_{v} U_{v}^{T} .
$$

Using (17) and (15), we finally have the approximation

$$
\mathcal{E}(Q[l]) \approx \Phi
$$

noting that

$$
\mathcal{E}(Q)=\left\{\Gamma \in \mathbb{R}^{3} \mid \Gamma^{T} Q^{-1} \Gamma \leq 1\right\} .
$$

One possible choice of $l$ yields the unique internal ellipsoid with maximum volume ${ }^{13}$ (the John ellipsoid), but this is not necessarily the best choice in terms of the flight dynamics. We can choose $l$ parametrically via some other means (for example, to emphasize a preferred maneuvering axis).

An example of the approximation of the AMS is shown in Figures 5 and 6. The boundary of $\Phi$ is formed from the moment span of a four-nozzle configuration with two large, high authority booster engines and two small core engines. The core engine constraints are elliptical while the booster constraints are circular. The modeled moment of inertia of the vehicle is $\operatorname{diag}\left(\left[\begin{array}{lll}1.09 & 3.76 & 4.83\end{array}\right]\right) \times 10^{8}$ slug $-\mathrm{ft}^{2}$; the engine parameters are given in Table 1 .

The true boundary has been found via the convex hull of a family of approximating ellipsoids ${ }^{12}$ while the single approximation is along only the direction $l=\left[\begin{array}{ccc}0 & 0 & 1\end{array}\right]^{T}$. The two-dimensional projection illustrates the complex shape of the boundary; where an intersection occurs, the boundary of the ellipsoid $\mathcal{E}(Q)$ is tangent to the manifold $\partial(\Phi)$.

\begin{tabular}{|c|c|c|c|c|c|c|}
\hline Engine & Type & \multicolumn{2}{|c|}{ Location (ft) } & Thrust (lbf) & Orientation & Angle Constraint \\
\hline \hline 1 & Booster & $r_{1}=\left[\begin{array}{lll}-87.2 & -20.7 & 0\end{array}\right]^{T}$ & $F_{1}=2.27 \times 10^{6}$ & $u_{1}=\left[\begin{array}{lll}1 & 0 & 0\end{array}\right]^{T}$ & $4^{\circ}$, circular \\
\hline 2 & Booster & $r_{2}=\left[\begin{array}{lll}-87.2 & +20.7 & 0\end{array}\right]^{T}$ & $F_{2}=2.27 \times 10^{6}$ & $u_{2}=\left[\begin{array}{lll}1 & 0 & 0\end{array}\right]^{T}$ & $4^{\circ}$, circular \\
\hline 3 & Core & $r_{3}=\left[\begin{array}{lll}-86.7 & -5.42 & 0\end{array}\right]^{T}$ & $F_{3}=4.63 \times 10^{5}$ & $u_{3}=\left[\begin{array}{lll}1 & 0 & 0\end{array}\right]^{T}$ & $5^{\circ}$ pitch, $7^{\circ}$ yaw, elliptical \\
\hline 4 & Core & $r_{4}=\left[\begin{array}{lll}-86.7 & +5.42 & 0\end{array}\right]^{T}$ & $F_{4}=4.63 \times 10^{5}$ & $u_{4}=\left[\begin{array}{lll}1 & 0 & 0\end{array}\right]^{T}$ & $5^{\circ}$ pitch, $7^{\circ}$ yaw, elliptical \\
\hline
\end{tabular}

Table 1. Example vehicle parameters 


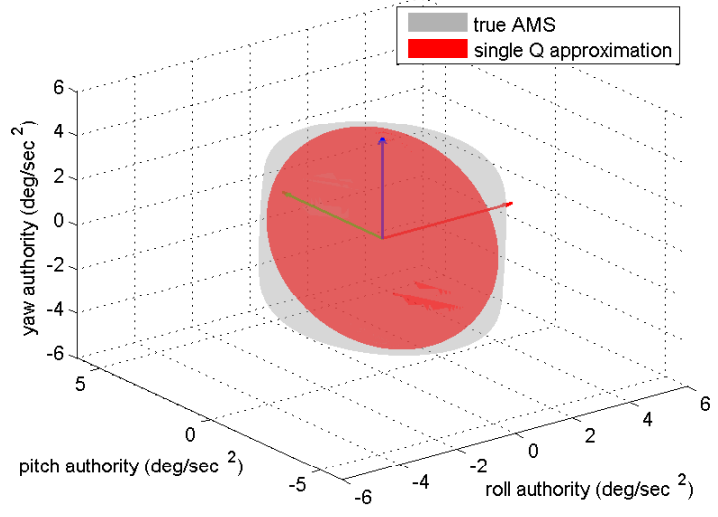

Figure 5. Ellipsoidal approximation of $\Phi$ (3-axis)

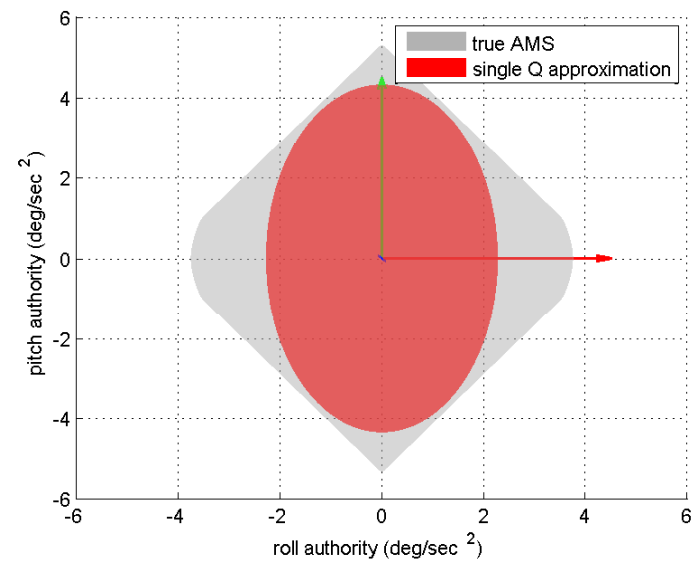

Figure 6. Ellipsoidal approximation of $\Phi$ (pitch-roll axes)

\section{E. Approximation of the AMS Under a Particular Inverse}

In order to choose an appropriate $P$, the geometry of $\Phi^{\star}$ must be determined so as to assign to each $\Phi^{\star}$ some order of merit. The ellipsoidal approximation (18) can be used to closely approximate the geometry of $\Phi^{\star}$.

Let $P$ be a particular generalized inverse that satisfies $B P=I$; partition $P$ as

$$
P=\left[\begin{array}{llll}
P_{1}^{T} & P_{2}^{T} & \ldots & P_{k}^{T}
\end{array}\right]^{T}
$$

and let $B_{i}^{\star}$ be a Moore-Penrose inverse such that $P_{i} B_{i}^{\star}=I$. Consider that there exists in $\mathbb{R}^{m}$ an image of $\mathcal{E}(Q)$ which can be partitioned into $k$ projections onto the constraint subspaces. Since $\mathcal{E}(Q)$ is an ellipsoid with center at the origin, its image under linear transformation must also consist of ellipsoids in $\mathbb{R}^{2}$ with centers at the origin. Suppose that the shape matrix of the image of $Q$ in each of $k$ subspaces is denoted by $R_{i}^{\star}$; we then have

$$
\xi_{i}^{\star}=\left\{\delta_{i} \in \mathbb{R}^{2} \mid \delta_{i}^{T} R_{i}^{\star-1} \delta_{i} \leq 1\right\}
$$

Noting that $\delta_{i}=P_{i} \Gamma$ and computing a suitable generalized inverse $B_{i}^{\star}=P_{i}^{\dagger}$, we again note that $\Gamma^{T} Q^{-1} \Gamma=\delta_{i}^{T} R_{i}^{\star-1} \delta_{i}$ and substitute $\Gamma=B_{i}^{\star} \delta_{i}$ to find that

$$
R_{i}^{\star-1}=B_{i}^{\star T} Q^{-1} B_{i}^{\star}
$$

The sets $\xi_{i}^{\star}$ and $\xi_{i}$, as a consequence of the aforementioned theorem, cannot be equal. Therefore, there exist two distinct cases. In the first case, the inverse map does not generate controls that violate the constraint boundaries. In this case, $\xi_{i}^{\star} \subset \xi_{i}$, and the ellipsoid $\mathcal{E}\left(R_{i}^{\star}\right)$ is an inclusion of $\mathcal{E}\left(R_{i}\right)$. In the second case, some portion of the inverse image lies outside the constraint boundary, and some lies within it (Figure 7). That portion that forms $\Phi^{\star}$ is the portion that is admissible, and therefore consists of the intersection $\xi_{i}^{\star} \cap \xi_{i}$ of two ellipsoids.

We have uncovered the complication that, to the accuracy of our foregoing approximation, while the total AMS $\Phi$ is the geometric sum of degenerate ellipsoids in $\mathbb{R}^{3}$, the particular AMS $\Phi^{\star}$ is in fact the geometric sum of, at most, the linear mapping of each of $k$ sets each formed by the intersection of two ellipsoids in $\mathbb{R}^{2}$. The latter geometry is non-trivial to compute, but it becomes clear that the intersections in $\mathbb{R}^{2}$ can be approximated to a high degree of accuracy via the use of another parametrized ellipsoid! In the trivial case of inclusion, the approximation is exact. Conveniently, the internal approximation of the intersection of two ellipsoids with zero center in $\mathbb{R}^{2}$ has a closed-form solution, ${ }^{14}$ given by

$$
\mathcal{E}\left(\hat{R}_{i}[l]\right)=\mathcal{E}\left(\frac{\left(R_{i}^{\star-1}-R_{i}^{-1}\right) l l^{T}\left(R_{i}^{\star-1}-R_{i}^{-1}\right)}{l^{T}\left(R_{i}^{\star-1}-R_{i}^{-1}\right) l}\right) .
$$

The determination of an inclusion versus an intersection is readily determined based on the sign of the eigenvalues of $\left(R_{i}^{\star-1}-R_{i}^{-1}\right)$. 


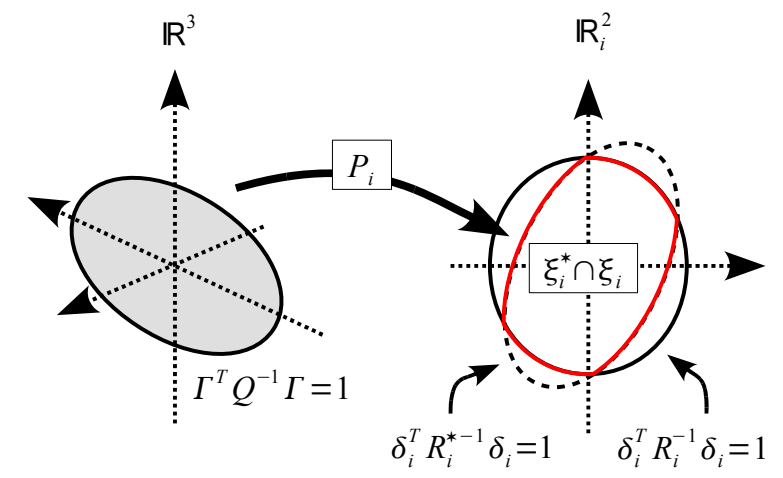

Figure 7. Topology of constraints under a particular inverse

Each new approximation $\mathcal{E}\left(\hat{R}_{i}\right) \approx \xi_{i}^{\star} \cap \xi_{i}$ can be mapped back into $\mathbb{R}^{3}$ using the original transformation $P_{i}^{\star}$, where

$$
Q_{i}^{\star-1}=P_{i}^{\star T} \hat{R}_{i}^{-1} P_{i}^{\star} .
$$

The ellipsoidal approximation $\mathcal{E}\left(Q^{\star}\right)$ to $\Phi^{\star}$ is then found from the Minkowski sum of the $\mathcal{E}\left(Q_{i}^{\star}\right)$, again using the same procedure (15-18).

An example of the ellipsoidal approximation of a particular inverse is shown in Figures 8 and 9 . The true boundary of $\Phi^{\star}$ is determined numerically as the convex hull of a set of points along unique directions on the unit ball, each determined via a bisection search to a small tolerance. The internal ellipsoidal approximation to $\Phi^{\star}$ is tight along the direction line $l=\left[\begin{array}{lll}0 & 0 & 1\end{array}\right]^{T}$.

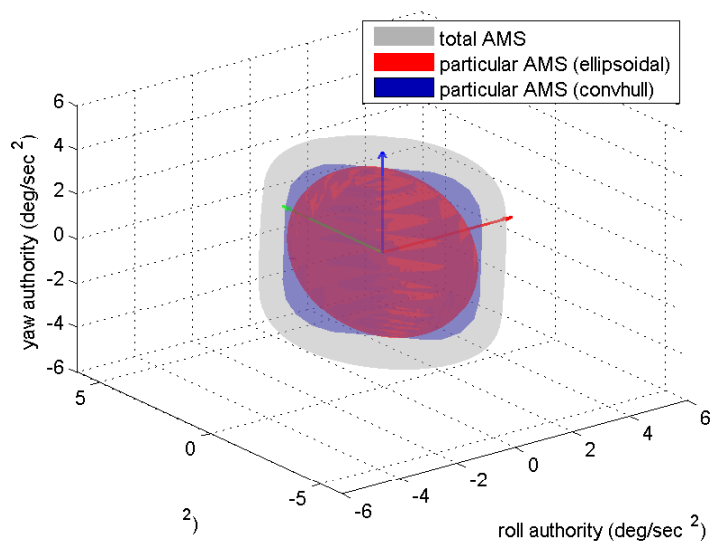

Figure 8. Ellipsoidal Approximation of $\Phi^{\star}$ (3-axis)

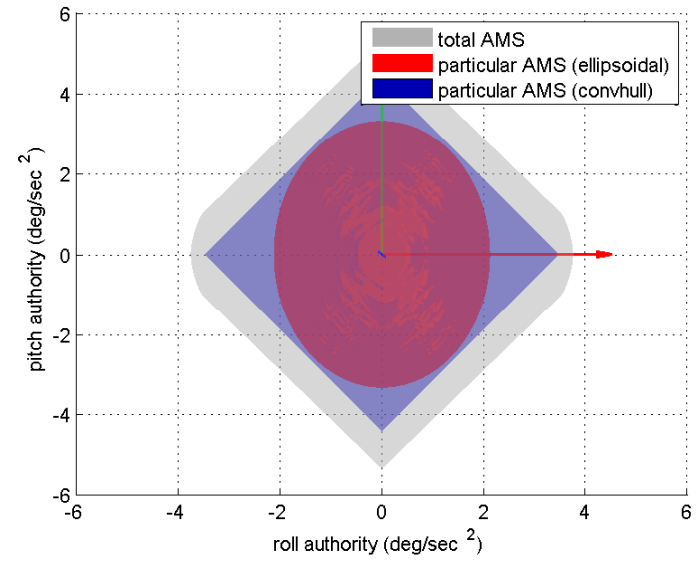

Figure 9. Ellipsoidal Approximation of $\Phi^{\star}$ (pitch-roll axes)

\section{Applications}

\section{A. Minimal Parameterization of the Weighted Generalized Inverse}

Through the use of a weighted least squares allocator, the number of free parameters in determining a generalized inverse that satisfies other optimality conditions can be considerably reduced, and this parameterized inverse can be optimized using the ellipsoidal approximation of the AMS.

There exists a unique solution that minimizes the weighted square of control deflection, ${ }^{6}$ given by the cost function

$$
H=\frac{1}{2} \Delta^{T} R \Delta+\lambda^{T}\left(\dot{\omega}_{c}-B \Delta\right)
$$


where $R \in \mathbb{R}^{2 k \times 2 k}$ is a symmetric, positive-definite matrix (analogous to the measurement covariance matrix in the least squares estimation dual problem) ${ }^{15}$ and $\lambda$ is a vector of Lagrange multipliers in the usual sense. We have the necessary conditions

$$
\begin{aligned}
& \nabla_{\Delta} H=R \Delta-B^{T} \lambda=0 \\
& \nabla_{\lambda} H=\dot{\omega}_{c}-B \Delta=0
\end{aligned}
$$

Letting the weight matrix $W=R^{-1}$, we solve (20) for $\lambda$ to find $\Delta=W B^{T} \lambda$; it follows from (21) that $\dot{\omega}_{c}-B W B^{T} \lambda=$ 0 and finally we arrive at the well-known weighted least squares solution

$$
\Delta=W B^{T}\left(B W B^{T}\right)^{-1} \dot{\omega}_{C}
$$

with the associated right generalized inverse

$$
P(W)=W B^{T}\left(B W B^{T}\right)^{-1},
$$

a variation of the Moore-Penrose generalized inverse. ${ }^{8}$

By constraining the selection of generalized inverses to the family of weighted least squares generalized inverses, the number of parameters is reduced. Without loss of generality, we may assume that for any measure of the optimality of $P$ (e.g. volume), the optimal weighting is diagonal and therefore has only $m=2 k$ parameters. In fact, given certain symmetry properties of the physical problem, the number of parameters may be further reduced to $k$. The argument again has an analog in estimation; the weight matrix can be diagonalized since there always exists an orthonormal transformation $T$ which expresses $B$ such that the constraint ellipse eigenaxes are aligned with the commanded degrees of freedom, and $\tilde{W}=T W T^{T}$. Note that this has already been loosely assumed in introducing the gimbal-to-body transformation $\left[T^{B G}\right]$ in equation (9), which also eliminates the redundant actuator degrees of freedom.

Illustrative of the value of the ellipsoidal approximation, as a simple example we compute the volume of the AMS as a function of a scalar-parameterized least squares inverse $P(\alpha)$. The parameter $\alpha$ is the relative weighting among the degrees of freedom associated with two solid rocket boosters (high thrust, large moment arm, and small angle constraints) and two core engines (low thrust, small moment arm, and large angle constraints), where $W=\operatorname{diag}(w)$ and

$$
w_{i}= \begin{cases}\alpha^{2}, & i=1 \ldots 4 \\ (1-\alpha)^{2}, & i=5 \ldots 8\end{cases}
$$

In Figure 10, the numerically-derived volume is compared with the log determinant of the inverse shape matrix of the approximation $\mathcal{E}\left(Q^{\star}\right)$. As anticipated, minimization of $\log \operatorname{det}\left(Q^{\star-1}\right)$ corresponds to simultaneous maximization of the volume of the associated parameterized AMS, enabling the rapid computation of a scalar cost function for more complex optimization routines involving a parameterized inverse dependent on multiple parameters.

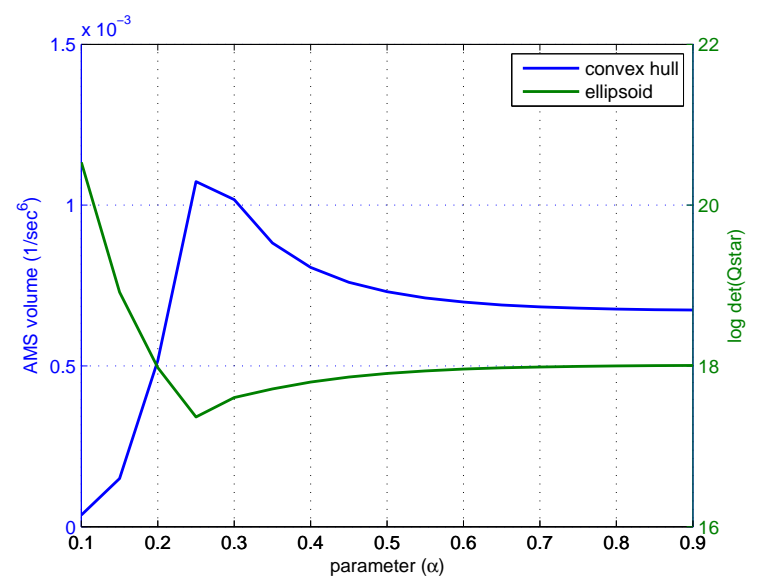

Figure 10. Approximation Correlation to Numerically-Derived Volume 


\section{B. Real-Time Steering Limiting}

Finally, we note one additional application of the approximation (18): in flight software it is often necessary to limit the commanded actuator deflection to a value less than the hardware limits so as to avoid commanding the actuators to the hardware stops which might result in servo damage or nozzle bell interference. With numerous actuator degrees of freedom and independent sets of constraints, a software limiting algorithm must separately compute, for example, each $\delta_{i}^{T} R_{i}^{-1} \delta_{i}$ and reduce individual deflection commands until all constraints are satisfied. Under these conditions, not only is the achieved angular acceleration $\Gamma \neq \dot{\omega}_{c}$, the collinearity condition is violated. Instead, in the case that $\dot{\omega}_{c}^{T} Q^{\star-1} \dot{\omega}_{c}>1$, the software algorithm must simply find a constant $k$ such that $\sqrt{k}\left(\dot{\omega}_{c}^{T} Q^{\star-1} \dot{\omega}_{c}\right)=1$, implying that

$$
k= \begin{cases}1, & \dot{\omega}_{c}^{T} Q^{\star-1} \dot{\omega}_{c} \leq 1 \\ \left(\dot{\omega}_{c}^{T} Q^{\star-1} \dot{\omega}_{c}\right)^{-2}, & \dot{\omega}_{c}^{T} Q^{\star-1} \dot{\omega}_{c}>1 .\end{cases}
$$

The scaled angular acceleration command $\tilde{\dot{\omega}}_{c}=k \dot{\omega}_{c}$ is then guaranteed not to saturate any actuator, and the achieved acceleration is in the same direction as that commanded.

\section{Discussion \& Conclusions}

In this paper we have developed and applied a direct, efficient mechanism for determining an accurate ellipsoidal approximation of the attainable moment set of a linear mapping of controls subject to a particular linear inverse and independent elliptical constraints. The formulation is well-suited to applications where elliptical constraints are more physically realistic than decoupled saturation constraints, especially with regards to the control of multi-actuated launch vehicles with vectored nozzles. While various nonlinear solutions of the associated constrained least-squares problem exist and can be obtained on-line algorithmically, the unique stability analysis requirements of flexible launch vehicles are better satisfied by a linear inverse. The present techniques allow an optimal parameterized linear inverse, such as a weighted least squares inverse, to be determined offline as a function of flight condition. Accompanied by an internal approximation of the boundaries of the attainable moments implemented as a steering limit algorithm, the present technique offers a unique and efficient mechanism for obtaining higher-performance thrust vector control.

While the methods presented herein are leveraged on rapid numerical analysis, this novel concept of determining the attainable moments subject to elliptical constraints has the potential to be extended to an analytic method of optimization. Currently, rapid approximations to the optimal weighted least squares solution, sufficiently precise to allow implementation in the flight software, can be determined for the special class of problems with circular constraints. However, a search for a closed-form approach that handles the general case is the subject of future work. Such algorithms are potentially beneficial for real-time adaptation to actuator failures and time-varying constraint boundaries. 


\section{References}

${ }^{1}$ W. Durham, "Constrained control allocation," J. Guidance, Control, and Dynamics, vol. 16, no. 4, pp. 717-725, 1993.

${ }^{2}$ W. Durham, "Efficient, near-optimal control allocation," J. Guidance, Control, and Dynamics, vol. 22, no. 2, pp. 369-372, 1998.

${ }^{3}$ A. B. Page and M. L. Steinberg, "A closed-loop comparison of control allocation methods," in AIAA Guidance, Navigation, and Control Conference and Exhibit, Denver, CO, AIAA, August 2000.

${ }^{4}$ J. Orr, M. Johnson, J. Wetherbee, and J. McDuffie, "State space implementation of linear perturbation dynamics equations for flexible launch vehicles," in AIAA Guidance, Navigation, and Control Conference, Chicago, Illinois, AIAA-2009-5962, 2009.

${ }^{5}$ J. Blakelock, Stability and Control of Aircraft and Missiles, 2nd Ed. Wiley-Interscience, 1991.

${ }^{6}$ R. E. Beck, Application of Control Allocation Methods to Linear Systems with Four or More Objectives. PhD thesis, Virginia Polytechnic Institute and State University, 2002.

${ }^{7}$ A. B. Kurzhanski and I. Valyi, Ellipsoidal Calculus for Estimation and Control. Birkhauser, 1996.

${ }^{8}$ R. Penrose, "A generalized inverse for matrices," Proc. Cambridge Phil. Soc., vol. 51, pp. 406-413, 1955.

${ }^{9}$ S. Campbell and C. Meyer, Generalized Inverses of Linear Transformations. Pitman, 1979.

${ }^{10}$ E. D. Buffington, J.M. and A. Teel, "Control allocation and zero dynamics," in Guidance, Navigation, and Control Conference, San Diego, CA, AIAA, July 1996.

${ }^{11}$ A. B. Kurzhanski and P. Varaiya, "Ellipsoidal techniques for reachability analysis: internal approximation," Systems $\mathcal{E}$ Control Letters, vol. 41 , no. 3, pp. $201-211,2000$.

${ }^{12}$ A. A. Kurzhanskiy and P. Varaiya, "Ellipsoidal toolbox," Tech. Rep. UCB/EECS-2006-46, EECS Department, University of California, Berkeley, May 2006.

${ }^{13}$ K. Ball, "Ellipsoids of maximal volume in convex bodies," Geometriae Dedicata, vol. 41, pp. 241-250, 1992.

${ }^{14}$ A. Y. Vazhentsev, "External ellipsoidal estimation of the union of two concentric ellipsoids and its applications," Computational Mathematics and Modeling, vol. 15, no. 2, pp. 110-122, 2004.

${ }^{15}$ J. L. Crassidis and J. L. Junkins, Optimal Estimation of Dynamic Systems. CRC Press, 2004.

\section{Acknowledgments}

This research was supported by the NASA Marshall Space Flight Center under contracts NNM06AA01Z and NNM08AA23T. The authors wish to thank our colleagues in the MSFC Flight Mechanics and Analysis Division for their numerous insights and contributions throughout the course of this work. In addition, the authors are grateful to A.A. Kurzhanskiy and P. Varaiya, whose Ellipsoidal Toolbox software was used in computing and visualizing many of the simulation results. 
Jeb Orr

Science Applications International Corporation John Wall

Dynamic Concepts, Inc.

Linear Approximation (10) Nozzless Writh Elliptical Constraints

AIAA Guidance, Navigation, and Control Conference, Portland, Oregon

August $9^{\text {th }}, 2011$ 
- Renewed interest in the development of large, new Heavy Lift Vehicles (HLV) has motivated research in the area of control allocation for systems with multiple rocket nozzles

- Rocket nozzle allocation can be treated as a trivial control allocation problem for simple, symmetric systems with symmetric constraints

- More complex geometries (e.g. Shuttle-derived) can benefit from an optimal allocator approach

- Example: least squares allocator; minimum control deflection

- We are concerned with handling general systems that have complex constraints and asymmetry
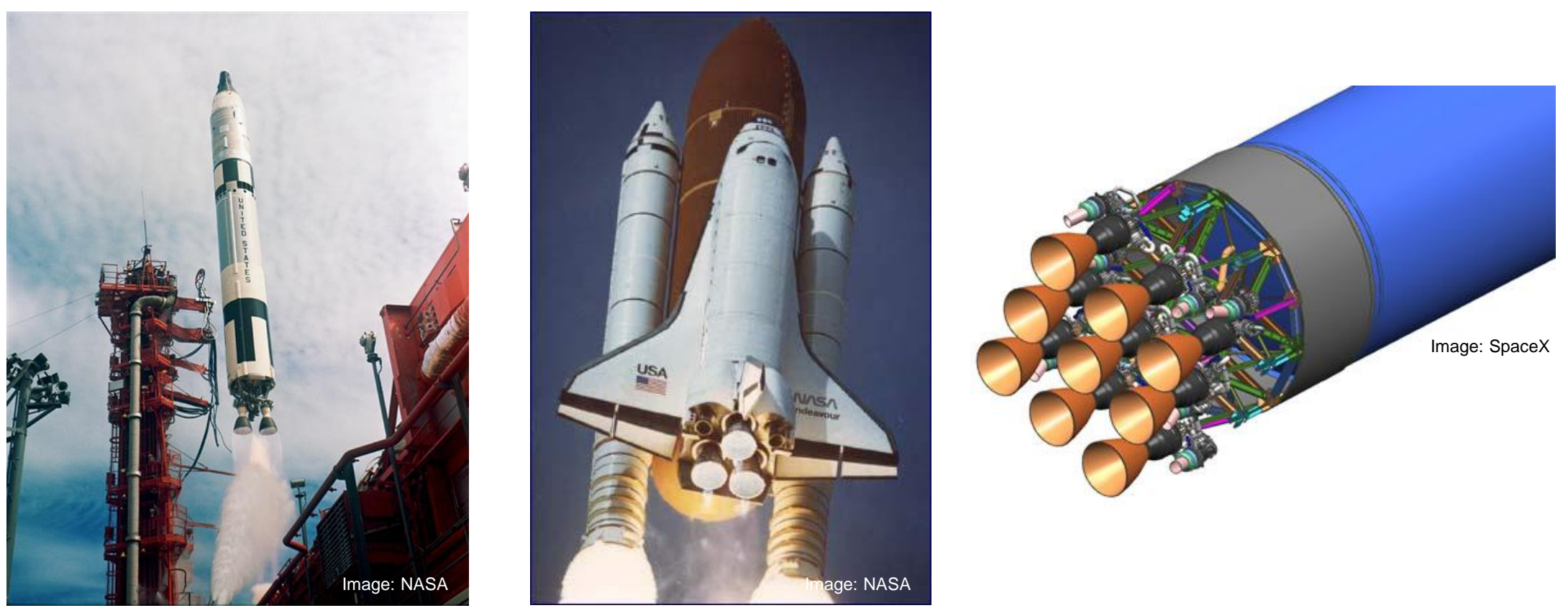
- Multi-actuated thrust vector controlled systems are well-posed for control allocation

- Typical redundant control authority in 3 axes with two or more nozzles

- Some configurations may have nine or more nozzles, each with two degrees of freedom

- Existing constrained solutions to the allocation problem exist and can be implemented online

- In the face of constraints, a potential solution are Constrained Least Squares (CLS) algorithms

- Often does not yield a moment collinear with command

- Requires online iteration/optimization

- Other constrained solutions include daisy chaining, etc.

- A nonlinear solution

- The constrained thrust vector control allocation problem differs from the aircraft problem

- Each control input has two degrees of freedom

- Saturation constraints are insufficient to represent the constraint boundary. Coupled constraints apply to two degrees of freedom each

- Due to significant servoelastic coupling, the choice of effector mixing at a given flight condition affects the stability of the closed-loop structural-dynamic system

- Linear allocators are preferred to enable linear stability analysis of the short period dynamics for flight certification

- A linear allocator can be computed online based on optimal parameterization (e.g. a weighting matrix) 
- Large booster thrust vector control (TVC) typically uses a pivoting nozzle with two actuators at 90 degree spacing about the undeflected thrust vector

- The geometric constraint boundary is approximately square, with round "corners" due to geometry of the actuator mechanism

- The two degrees of freedom of the actuator are arbitrarily aligned with the body axes; sometimes called "rock" and "tilt" (RSRMV) or "pitch" and "yaw" (SSME)

- We model the constraints conservatively as an ellipse, which is commonly used to size the thrust structure geometry

- Software angular limits on TVC travel are required to avoid actuator hard-stops (actuator damage), nozzle bell interference, launch structure contact, and plume impingement
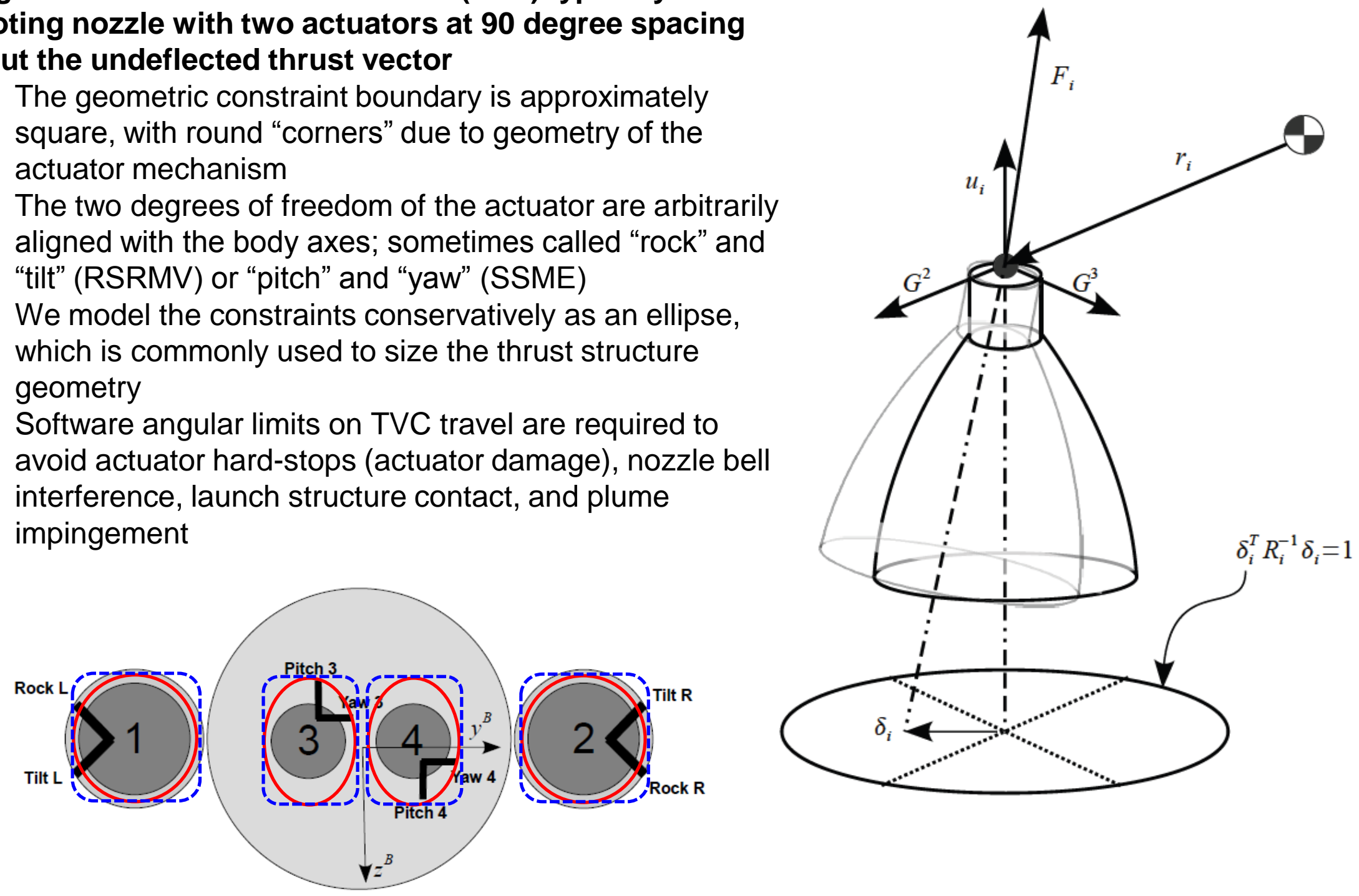
- The torque due to a single TVC deflection

$$
g_{i}=r_{i}^{\times}\left[T_{\theta}\right] F_{i} u_{i}
$$

- For small angles, is given by

$$
g_{i}=r_{i}^{\times} F_{i} u_{i}-F_{i} r_{i}^{\times} u_{i}^{\times} \theta_{i}
$$

- The total torque is then

$$
\begin{aligned}
g & =\sum_{i=1}^{k} r_{i}^{\times} F_{i} u_{i}-\sum_{i=1}^{k} F_{i} r_{i}^{\times} u_{i}^{\times}\left[T_{i}^{B G}\right] \delta_{i} \\
& =\sigma+M \Delta \\
& \quad \text { (residual + moment) }
\end{aligned}
$$

- The quantity

$$
\Delta=\left[\begin{array}{llll}
\delta_{1}^{T} & \delta_{2}^{T} & \cdots & \delta_{k}^{T}
\end{array}\right]^{T} \in \mathbb{R}^{2 k}
$$

is a control vector and each $\delta_{i} \in \mathbb{R}^{2}$ represents the two degrees of freedom for each effector. 
- We can write the torque in matrix form as

$$
\begin{aligned}
M & =\left[\begin{array}{llll}
M_{1} & M_{2} & \ldots & M_{k}
\end{array}\right]\left[T^{B G}\right] \\
& =\left[\begin{array}{llll}
-F_{1} r_{1}^{\times} u_{1}^{\times} & -F_{2} r_{2}^{\times} u_{2}^{\times} & \ldots & -F_{k} r_{k}^{\times} u_{k}^{\times}
\end{array}\right]\left[T^{B G}\right]
\end{aligned}
$$

- The applied angular acceleration in the linearized Euler equations is therefore

$$
\dot{\omega}=J^{-1} \sigma+J^{-1} M \Delta=\rho+B \Delta=\Gamma
$$

with the partitioning

$$
B=\left[\begin{array}{llll}
B_{1} & B_{2} & \ldots & B_{k}
\end{array}\right]
$$

- Assuming $\rho=0$ due to static cant or trim, we have the linear map

or equivalently

$$
\Gamma=B \Delta
$$

$$
\Gamma=\sum \gamma_{i} \quad \gamma_{i}=B_{i} \delta_{i}
$$




\section{Elliptical Constraints}

- Consider the constraint on each effector given by

$$
\delta_{i}^{T} R_{i}^{-1} \delta_{i} \leq 1, R_{i}=R_{i}^{T}>0
$$

so the admissible controls lie within the set

$$
\xi_{i}=\left\{\delta_{i} \in \mathbb{R}^{2} \mid \delta_{i}^{T} R_{i}^{-1} \delta_{i} \leq 1\right\}
$$

- The union of these sets yield $\Omega$, the set of admissible controls in $\mathbb{R}^{m}$.

- $\Omega$ has an image $\Phi$ under the transformation $B$.

- We refer to it as the attainable moment subset ${ }^{\star}$ (AMS).
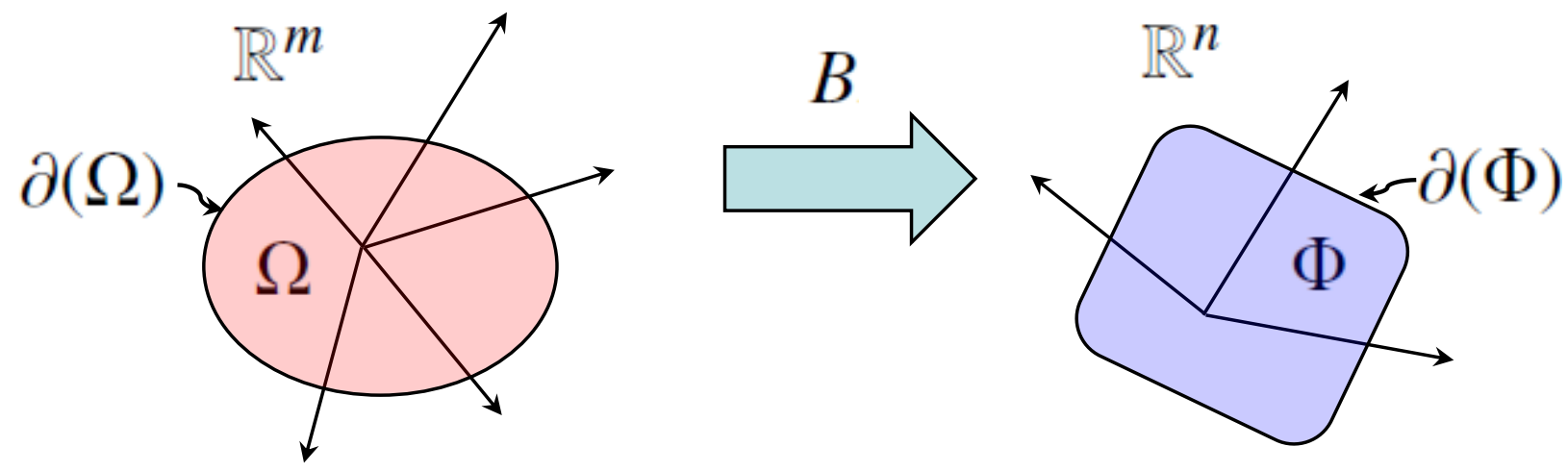

- There are only $\boldsymbol{n}$ directions in $\mathbb{R}^{m}$ that generate moments - all others generate local loads! 
- For each constraint ellipse $\mathcal{E}\left(R_{i}\right)$, we postulate that there exists its image in $\mathbb{R}^{3}$.

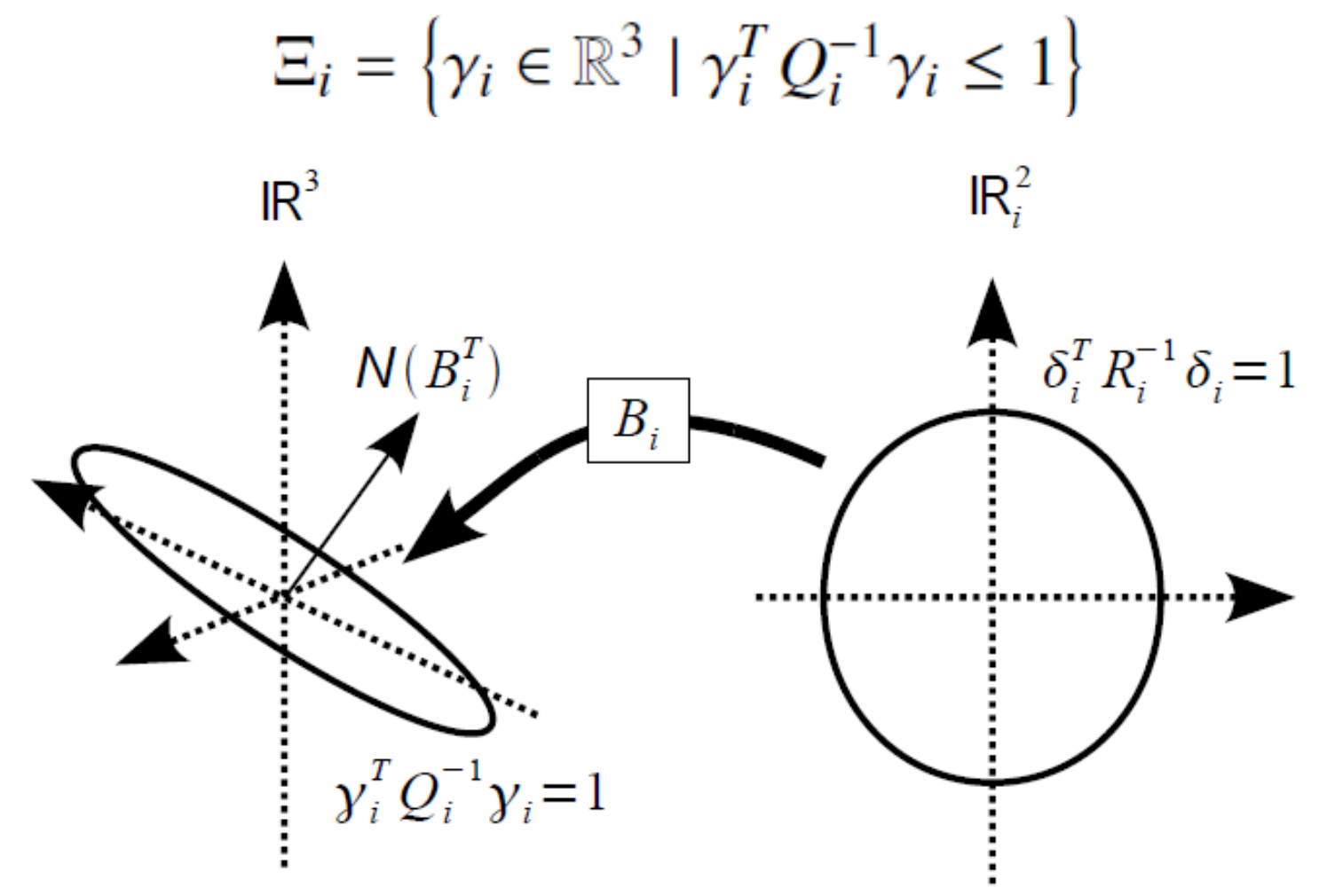

- We can solve for it directly! Given the Moore-Penrose inverse satisfying $B_{i} P_{i}^{\star}=I$,

$$
Q_{i}^{-1}=P_{i}^{\star T} R_{i}^{-1} P_{i}^{\star}
$$

- $\mathcal{E}\left(Q_{i}\right)$ is a degenerate ellipsoid in $\mathbb{R}^{3}$. 
- The true AMS, attainable under some (nonlinear) inverse, is found from the Minkowski sum of the images of $k$ degenerate ellipsoids;

$$
\Phi=\Xi_{1} \otimes \cdots \otimes \Xi_{k}=\bigcup_{\gamma_{1} \in \Xi_{1}} \cdots \bigcup_{\gamma_{k} \in \Xi_{k}}\left\{\gamma_{1}+\cdots+\gamma_{k}\right\}
$$

- The AMS is not, in general, an ellipsoid, but can be approximated by one (or many).
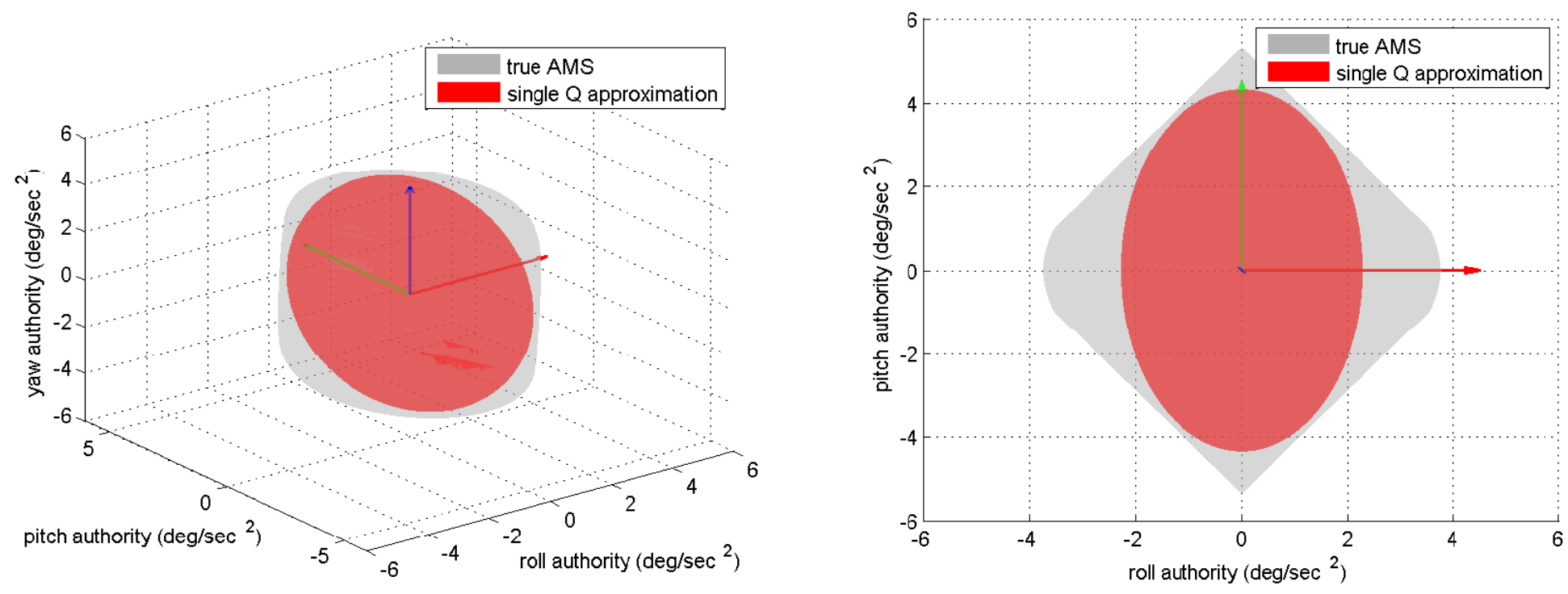
- We also define the particular AMS, that which is accessible under a particular linear inverse that satisfies

$$
B P=I
$$

such that

$$
\Delta=P \dot{\omega}_{c}
$$

- We refer to $P$ as an allocator or particular inverse.

- There are infinitely many $P$; the family of least-squares allocators parameterized by a weight matrix that minimizes

is often a good choice.

$$
H=\frac{1}{2} \Delta^{T} R \Delta \quad W=R^{-1}
$$

- There is no linear inverse that can access the entirety of the true AMS.

- In order to optimally parameterize the particular AMS to be "good", e.g., maximum volume, we must be able to determine its geometry

- The total or true AMS is relatively easy to find; the particular AMS is very difficult to find

- Must rely on a numerical method in the case of box/saturation constraints

- Numerical methods are expensive and difficult to use for optimization

- Using ellipsoidal approximations, we can solve for the particular AMS directly. 
- As a consequence of a linear inverse, some of the total AMS's image falls outside the constraint boundaries;

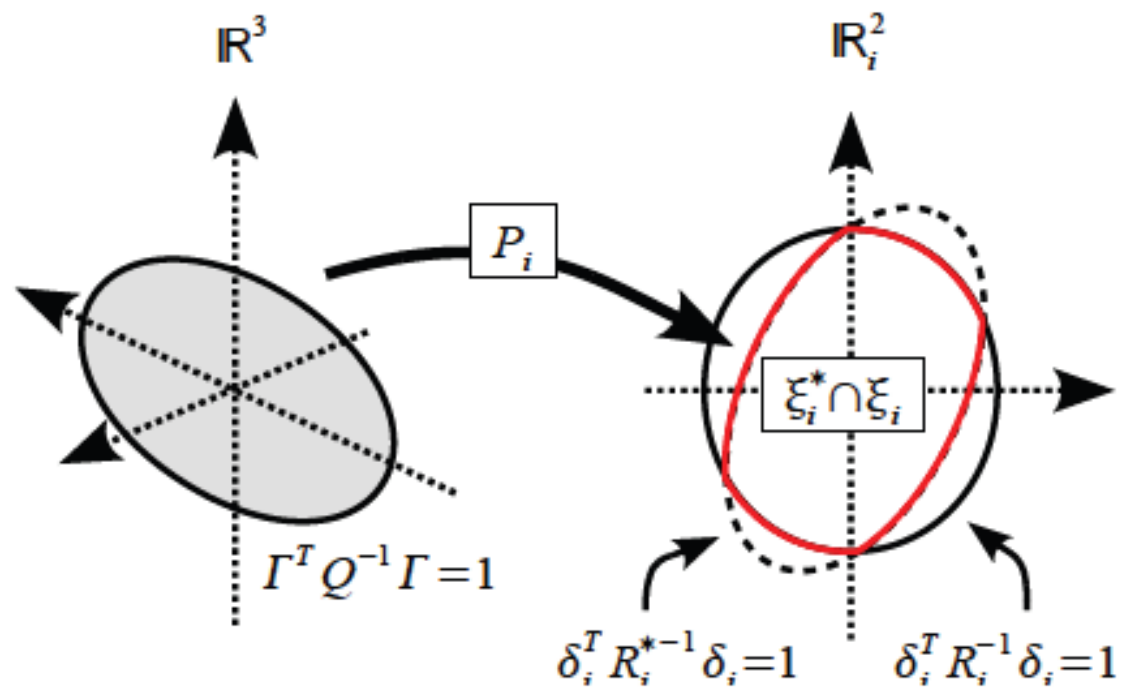

- The image of the intersection of these sets under $B$ forms the particular AMS $\Phi^{\star}$.

- Using an ellipsoidal approximation technique, we can find a very good internal approximation. 
- First, we determine the tight internal approximation of the total AMS $\mathcal{E}(Q[l]) \approx \Phi$

$$
\mathcal{E}(Q)=\left\{\Gamma \in \mathbb{R}^{3} \mid \Gamma^{T} Q^{-1} \Gamma \leq 1\right\}
$$

- The inverse image, or projection of $\mathcal{E}(Q)$ on the constraint boundaries is determined;

$$
\xi_{i}^{\star}=\left\{\delta_{i} \in \mathbb{R}^{2} \mid \delta_{i}^{T} R_{i}^{\star-1} \delta_{i} \leq 1\right\}
$$

- The internal approximation of the intersection $\xi_{i}^{\star} \cap \xi_{i}$ has a closed form solution given by

$$
\mathcal{E}_{-}\left(\hat{R}_{i}[l]\right)=\mathcal{E}\left(\frac{\left(R_{i}^{\star-1}-R_{i}^{-1}\right) l l^{T}\left(R_{i}^{\star-1}-R_{i}^{-1}\right)}{l^{T}\left(R_{i}^{\star-1}-R_{i}^{-1}\right) l}\right)
$$

(exempting inclusion, a trivial case)

- Finally, we map the intersection approximations back into $\mathbb{R}^{3}$ and find their geometric sum, using

$$
Q_{i}^{\star-1}=P_{i}^{\star T} \hat{R}_{i}^{-1} P_{i}^{\star}
$$


- The volume (log det) of the ellipsoidal approximation is correlated with the actual geometry, found (expensively) using a convex hull algorithm.
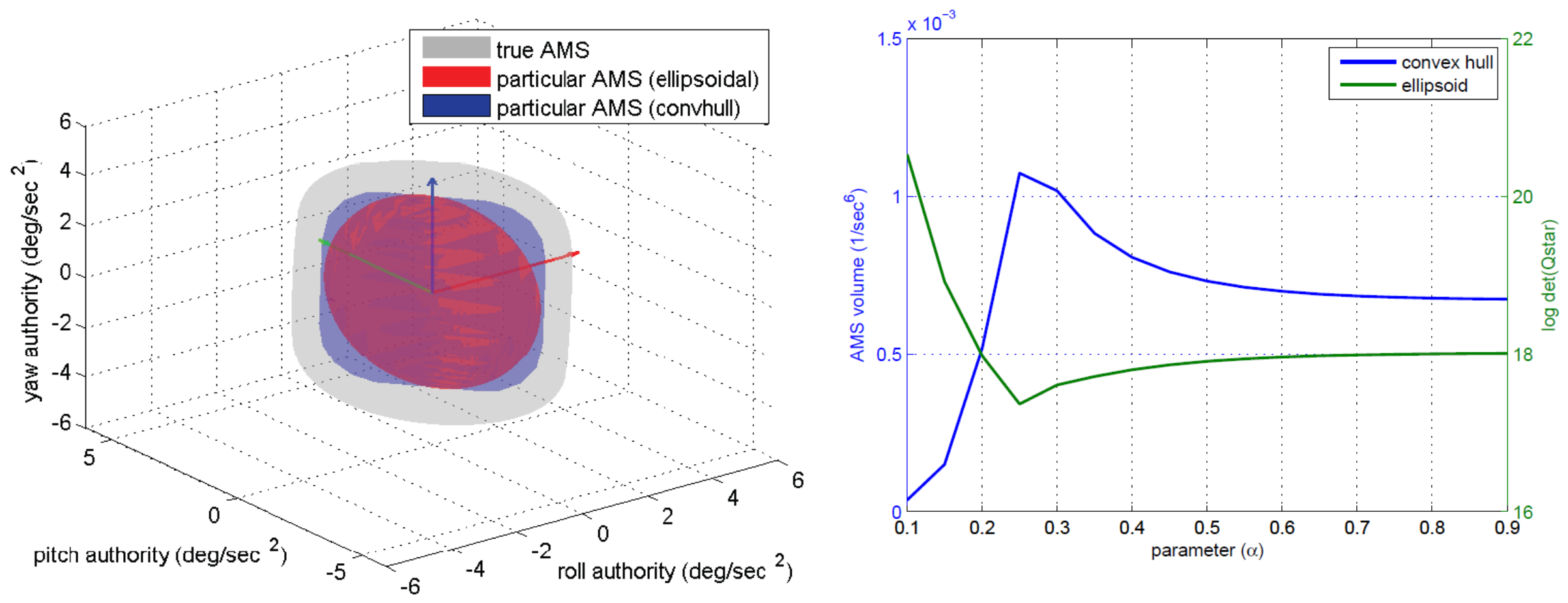

- The direct method can be used to optimize the volume of $\Phi^{\star}$ and find the best generalized inverse as a function of flight condition.

- The internal ellipsoid can also be used as a saturation limiter in the flight software. 
- A novel technique for determining the attainable moment subsets under linear mapping has been presented

- Exploits the special properties of elliptically constrained systems to avoid exhaustive numerical analysis to determine the geometry of the AMS

- Applications to launch vehicles with least-squares linear allocators yield optimally-parameterized inverses that maximize control authority and performance margin

- Most launch vehicles already use a special case of a least-squares allocator

- The present results generalize the theory somewhat for complex geometries and non-trivial cases with, for example, arbitrary cant angles

- Future work involves possible analytic maximum volume solutions to the least-squares allocator under certain conditions

- Possible extension to time-varying constraint boundaries for better real-time adaptation to degraded or failed actuators 